\title{
Could the Implantation of Dextranomer/Hyaluronic Acid Cause the Elevation of Neuron-Specific Enolase in Children Treated for Vesicoureteral Reflux?
}

\author{
Iva Bilić Čače ${ }^{1,2, \star}$, Ana Milardović ${ }^{1,2}$, Harry Nikolić ${ }^{3,4}$, \\ Ana Bosak Veršić ${ }^{3,4}$, Ivona Butorac Ahel ${ }^{1,2}$
}

\author{
${ }^{1}$ Pediatric Clinic, Clinical Hospital Centre \\ Rijeka, Croatia \\ ${ }^{2}$ Department of Pediatrics, University of \\ Rijeka, Faculty of Medicine, Rijeka, \\ Croatia \\ ${ }^{3}$ Department of Child Surgery, \\ University Hospital Centre Rijeka, Croatia \\ ${ }^{4}$ Department of Surgery, University of \\ Rijeka, Faculty of Medicine, Rijeka, \\ Croatia
}

\section{*Correspondence}

ivabiliccace@gmail.com

(Iva Bilić Čače)

\begin{abstract}
Background: Dextranomer/hyaluronic acid copolymer (Dx/HA) has been the most widely used bulking agent for the endoscopic treatment of vesicoureteral reflux. Case presentation: Here we report on a case of an 18-month-old male who underwent endoscopic treatment with Dx/HA for persistent high-grade unilateral vesicoureteral reflux (VUR). Three months later, due to the reappearance of contralateral VUR and indicated endoscopic treatment, a second cystoscopy was performed. A submucosal, well-vascularized mass localized on the left trigone and anteromedially to the left orifice was noted. Since it differed from the other similar implants previously detected on cystoscopy, it was misdiagnosed with a bladder tumor, indicating further evaluation. Extensive laboratory and imaging studies revealed normal findings, apart from high levels of neuron-specific enolase (NSE). A hypothesis made at that point was that the elevated level of NSE is due to the implantation of the Dx/HA. To test the hypothesis, two other healthy children previously treated with a Dx/HA implant were identified and their NSE levels measured. In both cases, the levels of NSE were elevated. Conclusion: The presented cases revealed that elevated levels of NSE are most likely due to a previous implantation of Dx/HA. This observation should be taken into consideration whenever there is a tentative diagnosis of bladder tumor following the endoscopic treatment of VUR with Dx/HA in a child.
\end{abstract}

\section{Keywords}

Dextranomer, Hyaluronic acid, Neuron-specific enolase, Bladder tumor

\section{Background}

Endoscopic treatment with dextranomer/hyaluronic acid copolymer $(\mathrm{Dx} / \mathrm{HA})$ is a feasible procedure and has become a reputable alternative to the surgery of ureteral re-implantation and long-term antibiotic prophylaxis in the treatment of children with vesicoureteral reflux (VUR) [1]. Since the implant is composed of dextranomer/ hyaluronic acid which remains in situ for up to 39 months, it is important for health care providers to be aware of the radiographic and endoscopic appearance of the implant during the follow-up period $[2,3]$. A bizarre radiographic appearance of implants may lead to a misdiagnosis of bladder tumor resulting in unnecessary and often invasive diagnostic evaluation [4].

Neuron-specific enolase (NSE) is produced by neuroendocrine tumors and may thus act as a tumor marker; it has been detected in patients with neuroblastoma, medullary thyroid cancer, carcinoid tumors, small cell lung tumors, melanoma, and endocrine tumors of the pancreas [5-7]. We report on a case of 18-month-old male who underwent endoscopic treatment with Dx/HA for persistent unilateral high-grade vesicoureteral reflux, misdiagnosed with bladder tumor three months later resulting in extensive diagnostic evaluation due to the high levels of NSE. 


\section{Case report}

An 18-month-old male was born with bilateral fetal hydronephrosis that was diagnosed at the 28 th week of gestation. The postnatal diagnosis was bilateral VUR, grade IV on the right side and grade $\mathrm{V}$ on the left. The urethra was normal with no signs of posterior urethral valves. At the age of 3 months, dynamic scintigraphy (MAG3) revealed parenchymal changes with differential renal function of $43 \%$ in the left kidney. He was treated with antibiotic prophylaxis (cefixim, co-trimoxasol) and followed up on a regular basis. During that period, his growth and development were satisfying and he had no urinary tract infections. At the age of 15 months, control voiding cystography showed persistent VUR only on the left side with deterioration of the left differential renal function (MAG3 $40 \%$ ). Endoscopic treatment with Dx/HA was performed. Control cystography three months later revealed reappearance of the right-sided VUR, so endoscopic treatment on the right side was indicated. During cystoscopy, prior to the planned implantation of Dx/HA at the right ureteral orifice site, an unexpected submucosal mass in the left half of the bladder was noted. It was described as a tumor, located on the left trigone and anteromedially to the left orifice. It measured approximately $1.5 \mathrm{~cm}$ in diameter and was not obstructing the orifice. The mucosa was intact, with a local hyperemic reaction. The edges were soft, the base wide. Being significantly remote from the left orifice, enlarged and unusually vascularized, it differed from the other similar implants previously detected on cystoscopy. These findings suggested further diagnostic evaluation.

Bladder ultrasound showed the right implant located just beneath the corresponding orifice (recently installed), while the other implant was located intravesically, significantly remote from the left ureteral orifice, projecting into the bladder cavity and unusually enlarged $(14 \times 10 \mathrm{~mm})$. A tentative diagnosis of a bladder tumor was made and extensive laboratory and imaging evaluation was initiated. Blood and urine analysis, including ferritin, lactate-dehydrogenase and specific tumor markers (serum carcinoembryonic antigen, human chorionic gonadotropin, alfa-1-fetoprotein, urine homovanillic acid, vanillylmandelic acid and normetanephrin) revealed that all the parameters were within reference values. However, the NSE was $39.7 \mu \mathrm{g} / \mathrm{L}$, which is more than two times higher than the reference values (normal reference < $16.3 \mu \mathrm{g} / \mathrm{L}$ ). Further imaging (magnetic resonance images of the thorax, abdomen and pelvis) excluded tumor expansion outside the bladder wall. Since laboratory and radiological evaluation showed no sign of tumor expansion outside the bladder, it was postulated that the tumorous growth might be associated with the previously implanted Dx/HA.

However, the significantly elevated level of NSE tumor marker was a "stumbling-stone" within our team. Another hypothesis made was that the elevated level of NSE is due to the implantation of Dx/HA. To test this hypothesis, the hospital record data were searched and two other healthy children (aged respectively 26 and 35 months) previously treated with Dx/HA implant were identified and their NSE levels evaluated. In both cases, all the urine and blood tests were normal except for the elevated levels of NSE, 17.3 and $28.5 \mu \mathrm{g} / \mathrm{L}$, respectively. Renal and bladder ultrasound confirmed the presence of the implants (less than $10 \mathrm{~mm}$ in size in both cases). Finally, the patient presented in this report was followed up for the subsequent 12 months and is now doing well. However, the NSE level remains elevated: it measured $31.3 \mu \mathrm{g} / \mathrm{L}$ a year after the procedure.

\section{Discussion}

$\mathrm{Dx} / \mathrm{HA}$ is a viscous gel that consists of non-animal-derived hyaluronic acid and dextranomer microspheres [8]. It is stabilized at the injection site by mild inflammatory response (a nonimmunogenic foreign body type of inflammatory response) [2]. It is well known that the hyaluronic acid component is degraded and replaced by a matrix of collagen, the implant volume decreasing over time by approximately $20 \%$. This can be visualized and measured by bladder ultrasound during regular follow-up as it remains in situ for up to 39 months $[2,4,8]$.

NSE, the neuronal form of the glycolytic enzyme enolase, is found almost exclusively in neurons and cells of neuroendocrine origin [9]. It is a dimeric form compounded of two $\gamma$ subunits that converts 2phosphoglycerate into phosphoenolpyruvate, measurable in blood and cerebrospinal fluid [10]. NSE is a known biomarker of ischemic brain damage. Its measurement is a valuable ancillary method for assessing the outcome after a traumatic brain injury, anoxic encephalopathy after cardiac arrest and stroke [9-11]. It has been proven to be elevated in patients with type 1 Diabetes mellitus [12]. As a tumor marker, NSE has been detected in patients with neuroblastoma, carcinoid tumors, medullary thyroid cancer, small cell lung tumor, endocrine tumors of the pancreas and melanoma [7]. It correlates well with the primary size of neuroblastoma and the outcome of the disease [13].

To the best of our knowledge, there is no previously reported case of elevated NSE associated with endoscopic treatment of VUR. Furthermore, to our knowledge, there are no published data that would demonstrate a potential correlation between VUR and increased NSE. In the case at hand, three months following the unilateral implantation of Dx/HA, its bulge was misdiagnosed as a tumorous growth, primary due to its unusual location within the bladder, its size and hypervascularization. In the author's (urologist) experience, it differed from other similar implants previously seen and/or described on cystoscopy. Regrettably, no biopsy of the lesion was performed at that point. Such misleading presentation of an unusual bladder growth was discussed further with other medical specialists and the consensus reached was that additional diagnostics should be done in order to exclude a potential malignant growth. This decision led to an extensive evaluation, causing significant anxiety to the patient and his family. An unexpectedly elevated level of NSE (tested as one of the tumor markers) 
extended the diagnostic algorithm, resulting in the exhaustion of medical resources and attributing to the overall cost increase. Given that all the other test results were normal, a conclusion was made that the elevated NSE level was likely to be associated with the implantation of Dx/HA. This hypothesis was confirmed on two other children previously treated with Dx/HA, whose NSE levels were also elevated. It is important to stress out that all the children were otherwise healthy, and none of them had other known likely reason for the elevated NSE. One might speculate on the possible role of infection causing the elevation of NSE, but a urinary tract infection had never been documented in these children during their regular follow-up. Furthermore, a quite recent study conducted on over 13,000 individuals with documented cystitis revealed that none of them had increased NSE [14]. The authors would like to stress out that the elevation of NSE in our case was an accidental finding resulting from an extensive diagnostic evaluation of an unsuccessful endoscopic treatment of VUR and the atypically distant migration of the implant, supported by two other cases of children previously treated with Dx/HA.

In conclusion, the presented cases revealed that elevated levels of NSE are most likely due to a previous implantation of Dx/HA. This observation should be taken into consideration when a tentative diagnosis of bladder tumor is made after endoscopic treatment of VUR with Dx/HA in a child. However, further studies are indicated before definitely correlating Dx/HA with high levels of NSE, as this may mislead the diagnostic process in patients whose elevated NSE levels are the result of a tumor.

\section{ACKNOWLEDGEMENTS}

We would like to express our sincere gratitude to Professor Mirna Šubat Dežulović, PhD, MD (retired) for providing guidance throughout this research.

\section{CONFLICT OF INTEREST}

The authors declare no conflict of interest.

\section{REFERENCES}

[1] Puri P, Mohanan N, Menezes M, Colhoun E. Endoscopic treatment of moderate and high grade vesicoureteral reflux in infants using dextranomer/hyaluronic acid. J Urol. 2009;81:1324-8.

[2] Stenberg A, Larsson E, Lackgren G. Endoscopic treatment with dextranomer-hyaluronic acid for vesicoureteral reflux: histological findings. J Urol. 2003;69:1109-13.

[3] Cerwinka WH, Qian J, Easley KA, Scherz HC, Kirsch AJ. Appearance of dextranomer/hyaluronic Acid copolymer implants on computerized tomography after endoscopic treatment of vesicoureteral reflux in children. J Urol. 2009;181:1324-8.

[4] Decaro JJ, Kirsch AJ. Dextranomer-hyaluronic Acid implants misdiagnosed as bladder tumor on transvaginal ultrasonography. Obstet Gynecol. 2012;119:476-8.

[5] Kaiser E, Kuzmits R, Pregant P, Burghuber O, Worofka W. Clinical biochemistry of neuron specific enolase. Clin Chim Acta. 1989;183:13-31.

[6] Oberg K, Janson ET, Eriksson B. Tumour markers in neuroendocrine tumours. Ital J Gastroenterol Hepatol. 1999;31:160-2.

[7] Tapia FJ, Polak JM, Barbosa AJ, Bloom SR, Marangos PJ, Dermody $\mathrm{C}$ et al. Neuron-specific enolase is produced by neuroendocrine tumours. Lancet. 1981;1:808-11.

[8] Greenbaum LA, Mesrobian HGO. Vesicoureteral reflux. Pediatr Clin N Am. 2006;53:413-427.

[9] Kirino T, Brightman MW, Oertel WH, Schmechel DE, Marangos PJ. Neuron-specific enolase as an index of neuronal regeneration and reinnervation. J Neurosci. 1983;3:915-923.

[10] Rech TH, Rios Vieira SR, Nagel F, Brauner JS, Scalco R. Serum neuron-specific enolase as early predictor of outcome after in-hospital cardiac arrest: a cohort study. Crit Care. 2006;10:R133.

[11] Guzel A, Er U, Tatli M, Aluclu V, Ozkan U, Duzenli Y, et al. Serum neuron-specific ebnolase as a predictor of short-term outcome and its correlation with Glasgow Coma scale in traumatic brain injury. Neurosurg Rev. 2008;31:439-44.

[12] Elshorbagy HH, Barseem NF, Elsadek AE, Alsohokary AH, Maksoud YHA, Abdulsamea SE, et al. Serum Neuron-specific Enolase and S100 Calcium-binding Protein B in Pediatric Diabetic Ketoacidosis. J Clin Res Pediatr Endocrinol. 2019;11:374-387.

[13] Georgantzi K, Skoldenberg EG, Stridsberg M, Kogner P, Jakobson A, Tiensuu Janson E, et al. Chromogranin A and neuron-specific enolase in neuroblastoma: Correlation to stage and prognostic factors. Pediatr Hematol Oncol. 2018;35:156-165.

[14] Could Cystitis cause Neuron-specific enolase increased, in real world? 2018. accessable on: http://www. ehealthme.com/cs/ cystitis/neuron-specifictenolase+increased.

How to cite this article: Iva Bilić Čače, Ana Milardović, Harry Nikolić, Ana Bosak Veršić, Ivona Butorac Ahel. Could the Implantation of Dextranomer/Hyaluronic Acid Cause the Elevation of Neuron-Specific Enolase in Children Treated for Vesicoureteral Reflux? Signa Vitae. 2020;16(1):183-185. doi:10.22514/sv.2020.16.0025. 